\title{
Tratamientos térmicos de hormigones
}

\section{GENERALIDADES}

La rapidez de ejecución es una necesidad de rango económico que obliga hoy en día a la búsqueda de nuevas soluciones.

Rapidez, precisión, calidad y economía, son cuatro imperativos para el empresario o constructor de edificios, que parecen conseguirse con la utilización del hormigón como materia única para muros de carga y forjados. Indudablemente cualquier factor que permita mejorar alguna o varias de estas magnitudes sin perturbar a las restantes, será bien acogido. Esto ha permitido crear nuevas técnicas para el tratamiento del hormigón, como es el conseguir un fraguado rápido.

En un principio el tratamiento térmico del hormigón era el medio que impedía la congelación del agua en climas muy fríos, tan perjudicial para el fraguado del mismo. Puede advertirse cómo esta técnica no nace por su fin actual, sino como un medio de prevención y seguridad contra mayores perjuicios.

Posteriormente ha podido advertirse cómo el tratamiento térmico del hormigón o de sus componentes, además de un recurso contra las heladas, es en toda época del año un medio de acelerar el fraguado y, por tanto, una nueva herramienta que, junto con los aditivos para fraguado rápido, permiten nuevas economías en la edificación considerada como una industria.

En la documentación consultada hemos podido advertir cómo, por diferentes causas, realmente existe una técnica de endurecimiento del hormigón por calefacción eléctrica.

El resultado general de estos estudios, desde un punto de vista práctico, sera el conseguir un fraguado tal del hormigón que permita desencofrar en breves horas; esto es, que el tiempo máximo requerido para el endurecimiento del hormigón sea el de descanso nocturno del personal. Este resultado permitirá incrementar la productividad de las obras en general, acelerar la rotación de moldes y encofrados y, por último, disminuir la duración total de la obra, con los consiguientes beneficios para el empresario de reducción de gastos generales imputados, reducción de alquileres o amortización de equipo y maquinaria, etc... 


\section{TECNICAS DE TRATAMIENTO TERMICO DE HORMIGONES}

\section{DISTINTOS SISTEMAS}

El tratamiento térmico de hormigones como medio de endurecimiento rápido comenzó a utilizarse principalmente en bloques y elementos prefabricados y en la fabricación de vigas pretensadas. Las primeras aplicaciones industriales de endurecimiento eléctrico del hormigón fueron emprendidas en 1958 en Francia, en una fábrica de Toulouse, que producía viguetas pretensadas de hormigón y cerámica.

Posteriormente se aplicó en las propias obras para el fraguado rápido del hormigón colado entre encofrados, para muros y forjados.

A continuación vamos a referirnos a los distintos métodos que pueden utilizarse haciendo una breve referencia sobre cada uno de ellos:

\section{Sistemas eléctricos}

a) Calefacción desde el interior de la propia masa:

1. ${ }^{\circ}$ Conductibilidad eléctrica de la pasta del cemento.

Este sistema, basado en la hiberación de calor por efecto Joule en la masa del hormigón, fue preconizado en Suecia antes de la guerra, y ha sido utilizado sobre todo desde 1940 en la U.R.S.S., con el fin de acelerar la construcción de centrales en el Ural. A veces se ha mejorado la conductibilidad eléctrica del hormigón añadiendo al cemento pequeñas cantidades de ácidos o de sales, que por otro lado son altamente perjudiciales para el mismo. Unos electrodos situados en la periferia de los macizos se conectan a una fuente de energía de baja tensión de frecuencia industrial.

2. ${ }^{\circ}$ Conductibilidad eléctrica del encofrado.

Como consecuencia de trabajos efectuados a partir de 1958, a veces es más económico servirse de las armaduras metálicas que utilizar resistencias eléctricas. Los primeros resultados obtenidos con este método se presentaron al Congreso de la Oficicina Internacional del Hormigón Manufacturado que se celebró en Estocolmo en 1960. En nuestros días se tratan cotidianamente más de $30 \mathrm{~km}$ de vigas para suelos, de cerámica y hormigón pretensado.

3. ${ }^{\circ}$ Resistencias eléctricas aisladas sumergidas y perdidas.

Para piezas no armadas, o cuando no se pretende el empleo de las armaduras como resistencias eléctricas, puede ser interesante recurrir a un calentamiento con la ayuda de hilos conductores aislados, sumergidos en la pasta cuando se confeccionaron las piezas, y que se pierden. Este nuevo procedimiento se presentó en 1963 al B.B.I.M., de París, y al Simposio que se celebró en Moscú, en julio de 1964. Desde 1962 ha sido objeto en Europa de numerosas aplicaciones.

Por ejemplo en las viguetas para forjado de pisos, una potencia por m.l. de $150 \mathrm{~W}$ se traduce en una resistencia a la compresión de alrededor de los $250 \mathrm{kp} / \mathrm{cm}^{2}$ a la mañana siguiente. Seis o siete fábricas utilizando este método funcionan actualmente en Francia, una de alrededor de $5.000 \mathrm{~kW}$ en Bélgica y otra de $1.500 \mathrm{~kW}$ en Portugal. El precio de coste total del endurecimiento eléctrico es del orden del 2,5 $\%$ del precio de venta del forjado. 
Esta técnica, da igualmente buenos resultados contra el hielo que como procedimiento en la aceleración del endurecimiento. Para grandes vigas o jácenas pretensadas, especialmente en las zonas de anclaje, se pueden obtener resistencias del orden de 80 a $300 \mathrm{kp} / \mathrm{cm}^{2}$ al cabo de unas 12 horas del vertido.

El rendimiento es bueno cuando se trata de placas de un espesor de unos 15 a $20 \mathrm{~cm}$, pero resulta un poco caro para las delgadas, del orden de 5 a $7 \mathrm{~cm}$, pues la separación entre la resistencia es siempre más o menos la misma; por lo tanto, una base de $5 \mathrm{~cm}$ utiliza, por $\mathrm{m}^{3}$ de hormigón, 4 veces más de resistencias, $\mathrm{y}$ por lo tanto de $\mathrm{kW} / \mathrm{h}$, que una de $20 \mathrm{~cm}$ de grueso.

4. ${ }^{\circ}$ Calentamiento dieléctrico.

Este procedimiento permanece por el momento en estado experimental. No obstante, en laboratorio ya se han obtenido resultados satisfactorios. La aceleración del endurecimiento por calentamiento dieléctrico está siendo actualmente considerada, sólo o asociada con otro modo de calentamiento, en particular en la prefabricación de tableros que contienen en su interior aislantes térmicos o fónicos.

b) Calefacción desde el exterior:

$\left.1^{\circ}\right)$ Moldes de hormigón calefactores.

Este procedimiento es especialmente interesante cuando el peso de los cuerpos calefactores es indiferente, pues éstos llegan a pesar aproximadamente $60 \mathrm{kp} / \mathrm{m}^{2}$, lo que sería excesivo para los moldes que deben elevarse, como por ejemplo las mesas calefactoras.

Comercialmente existen en forma de una losa cuadrada de hormigón, de $30 \times 30$ $\mathrm{cm}$, con dos salidas eléctricas aisladas que permiten colocarlas en serie y fijarlas, ya sea a los moldes, ya sea para construir suelos calefactores, muy interesantes cuando hace frío, en los andenes y sitios donde el público espera.

2.) Moldes metálicos calefactores.

Utilizados preferentemente en grandes elementos prefabricados, como puedan ser aquéllos empleados para forjados de pisos. En este caso el elemento calefactor utilizado queda en contacto con el molde metálico, produciéndose la transmisión del calor a través del mismo.

3.') Encofrados con contrachapado calefactor.

El encofrado se realiza en este caso con dos contrachapados en forma de "sandwich" que contienen en su interior una malla calefactora también por efecto Joule. En Francia se han obtenido resistencias de 100 a $120 \mathrm{kp} / \mathrm{cm}^{2}$ para placas de $15 \mathrm{~cm}$ de espesor.

Indudablemente el coste del encofrado es superior al de otro tipo normal, pero es posible amortizarlo en un número de utilizaciones superior a 200 coladas.

4. ${ }^{\circ}$ Mantas calefactoras.

Pueden emplearse también mantas o coberturas calefactoras, con un aislamiento adecuado en la parte superior, que permiten su instalación en obras sin mayor trabajo que colocar directamente la cobertura sobre el hormigón colado.

Estas mantas pueden usarse también como preventivos para las heladas. 


\section{Sistemas de vapor}

En el estudio del endurecimiento rápido del hormigón es necesario tener en cuenta el fenómeno psicrométrico que aparece en la capa libre del hormigón colado.

Durante el fraguado normal se produce una evaporación de agua que posteriormente es necesario reponer; si se produce un tratamiento térmico adicional, la temperatura del agua contenida en el hormigón aumentará, y por tanto con ella su presión de vapor. La diferencia entre esta presión de vapor y la presión parcial del vapor de agua contenido en el aire ambiente aumentará, creciendo por consiguiente la evaporación. El resultado es obvio; al calentar el hormigón la evaporación del agua se incrementa.

Puede evitarse esta pérdida de agua de dos formas distintas: el camino tradicional consiste en regar el hormigón colado; también puede recubrirse la pasta con una lona o manta impermeable, creando encima del hormigón una atmósfera saturada que impida la evaporación del agua, pero las pérdidas por infiltración nunca podrán ser evitadas.

Si además se aporta a esta atmósfera saturada agua en forma de vapor en cantidad comparable a las pérdidas de infiltración y según las necesidades del hormigón para un fraguado adecuado, el problema psicrométrico habrá desaparecido.

Por otro lado, la aportación de vapor supone también un aporte térmico adicional que permitirá equilibrar la temperatura del hormigón en sus dos caras.

En la práctica pueden presentarse varias modalidades:

- Autoclave

La pasta se introduce en un recipiente de acero que se encuentra a temperaturas entre los $120^{\circ}$ y $190^{\circ} \mathrm{C}$ y bajo una presión absoluta comprendida entre los 2 y $13 \mathrm{kp} /$ $/ \mathrm{cm}^{2}$ de vapor saturado. Este tratamiento limita la calefacción necesaria a un período muy breve, del orden de las 3 a 4 horas. Con 12 horas de tratamiento se obtienen generalmente hormigones de resistencias muy próximas a la máxima, incluso ha sido superada en U.S.A., debido a la reacción puzolánica o sílico-cálcica del hormigón utilizado. Sólo puede emplearse este sistema en la fabricación de pequeñas piezas.

\section{- Generador de vapor}

La aplicación de los estudios del comportamiento de probetas sometidas a autoclaves ha permitido la utilización de estas técnicas en obra valiéndose de pequeños generadores de vapor totalmente compactos.

\section{Sistemas de calefacción previa}

En otros casos es práctica usual calentar los elementos componentes del hormigón antes de su colada, particularmente el agua y la arena. Este sistema suele ser conveniente en la producción de elementos prefabricados, en donde el tiempo y el espacio que media entre el calentamiento y el fraguado es corto. En obras de construcción, por el contrario, las pérdidas que se producen hasta que el hormigón es colado hacen perder todo interés por este sistema.

En la práctica pueden presentarse distintas modalidades. 
a) Agua caliente

El tratamiento por agua caliente a la temperatura de $60^{\circ}$ a $80^{\circ} \mathrm{C}$ precisa normalmente de 8 a 12 horas para la obtención de piezas con resistencias convenientes. Es pues menos eficaz que el tratamiento a vapor, y la pérdida de resistencia a los 28 días es función de la temperatura final del hormigón y de la temperatura de punto de rocío del medio ambiente.

b) Calefacción de la arena

A pesar de que por este medio se han conseguido mejores resultados que por el anterior, el hecho es que no puede considerarse este sistema como adecuado para una obra.

\section{Calefacción por aire caliente}

En muchos casos resulta más económico calentar el hormigón mediante aire caliente. En obras de construcción esto es posible cerrando temporalmente el local, cuyos muros y forjado de techo recién colados están todavía encofrados, e inyectando aire caliente mediante generadores de aire que consuman gas o fuel-oil.

\section{Estufado}

Consiste en introducir la pasta después de amasada y de un período de espera en una estufa, para ser sometida durante 4 a 10 horas a una temperatura entre 10 s $70^{\circ}$ y $80^{\circ} \mathrm{C}$. Esta operación permite obtener una resistencia a la compresión parecida a la que se obtendría al cabo de 7 días de endurecimiento natural.

Este sistema sólo puede considerarse como instrumento de investigación en laboratorio o para la fabricación de elementos prefabricados.

\section{Conclusión}

Puede advertirse cómo existen numerosos caminos a seguir, condicionados en la práctica, por su vialidad económica. Realizaremos un estudio técnico previo para posteriormente conocer la realidad de coste de cada combustible. En la práctica también podrán utilizarse simultáneamente dos o más sistemas de los descritos.

\section{TEORIA GENERAL}

Como hemos ya indicado, tras los estudios de S. A. Mironov en Rusia y de C. Martinet en Francia se ha llegado a una verdadera técnica de tratamiento térmico de hormigones mediante la electricidad. Es debido a esto por lo que en el siguiente estudio técnico nos referimos muchas veces a este medio de calefacción, aunque no supone una selección del mismo "a priori". 


\section{Variación de la composición química}

Ha podido observarse, en el caso del cemento portland, que los hidratos que se forman con el calor, a presión atmosférica, no difieren en absoluto de los formados a temperatura normal. Mediante la utilización de análisis con rayos X pudo llegarse a este extremo, ya que no aparecían formaciones cristalinas $u$ otras estructuras que pudieran ser perjudiciales a la resistencia eléctrica del hormigón. Incluso cementos procedentes de la escoria de altos hornos se comportan de la misma forma y únicamente los cementos especiales -aluminosos, sobresulfatados o rápidos, etc.- pueden presentar alguna anormalidad al ser tratados térmicamente.

Indudablemente existen temperaturas a partir de las cuales estas afirmaciones no son correctas; precisamente en este punto, esto es, en la determinación de la temperatura máxima permisible, es en donde hoy en día existen ciertas discrepancias.

En el caso de emplear la conductibilidad eléctrica de la pasta se pueden presentar fenómenos de corrosión de la armadura o bien una porosidad excesiva producida por la ligera electrólisis creada por la presencia de sales, aunque sean en trazos infinitesimales. Por ejemplo, la electrólisis del aluminoferrito $\left(\mathrm{Al}_{2} \mathrm{O}_{3} \cdot \mathrm{Fe}_{2} \mathrm{O}_{3} \cdot 4 \mathrm{CaO}\right)$ produce particularmente una oxidación anódica. Se producen también en estos casos fenómenos de difusión de hidrógeno atómico.

\section{Determinación de la potencia calorífica necesaria}

En primer lugar, y antes de calcular la potencia calorífica necesaria, es necesario determinar el objetivo deseado: bien un tiempo de desencofrado (D) o bien una resistencia mecánica $(\mathrm{R})$ determinada.

A su vez, en cada caso pueden presentarse distintas soluciones: desencofrado rápido (DR) - del orden de una hora- o desencofrado lento (DL) - hasta una noche-, o bien, resistencia elevada $(\mathrm{RH})$, resistencia parcial (RP) para transportar las piezas por ejemplo y resistencia localizada (RL), lo que puede representar una variación de la resistencia deseada de 70 a $400 \mathrm{kp} / \mathrm{cm}^{2}$.

En nuestro caso fijaremos como objetivo la posibilidad de desencofrar en un máximo de 8 horas. En la figura 1 pueden apreciarse las curvas de consumo en $\mathrm{kW}-\mathrm{h} / \mathrm{t}$ de hormigón diseñadas por C. Martinet, en función del coeficiente de Módulo, definiendo como tal al cociente entre la superficie enfriada por m.l. y la sección de la pieza:

$$
\text { Coeficiente de Módulo }=\frac{\text { Superficie enfriada por m.l. }\left(\mathrm{en}^{2} / \mathrm{m}\right)}{\sec \text { ) }} \text {. }
$$

Puede apreciarse en esta figura cómo crece el consumo de energía por tonelada de hormigón al aumentar el tipo de Módulo, ya que la superficie enfriada aumenta proporcionalmente. Esta curva debe aplicarse preferentemente al cálculo de potencia calorífica necesaria en la prefabricación de vigas y tuberías.

Puede calcularse la resistencia a la compresión del hormigón recién colado mediante la ayuda de la curva de Nurse-Saul, que facilita esta magnitud en función del factor de madurez. 


\section{CURVAS DE CONSUMO}

Curvas de consumo en kW-h/t de hormigón en función del módulo " $\mathrm{M}$ " de la pieza.

$$
\mathrm{M}=\frac{\text { Superficie de enfriamiento por m.l. }}{\text { Sección de la pieza }}
$$

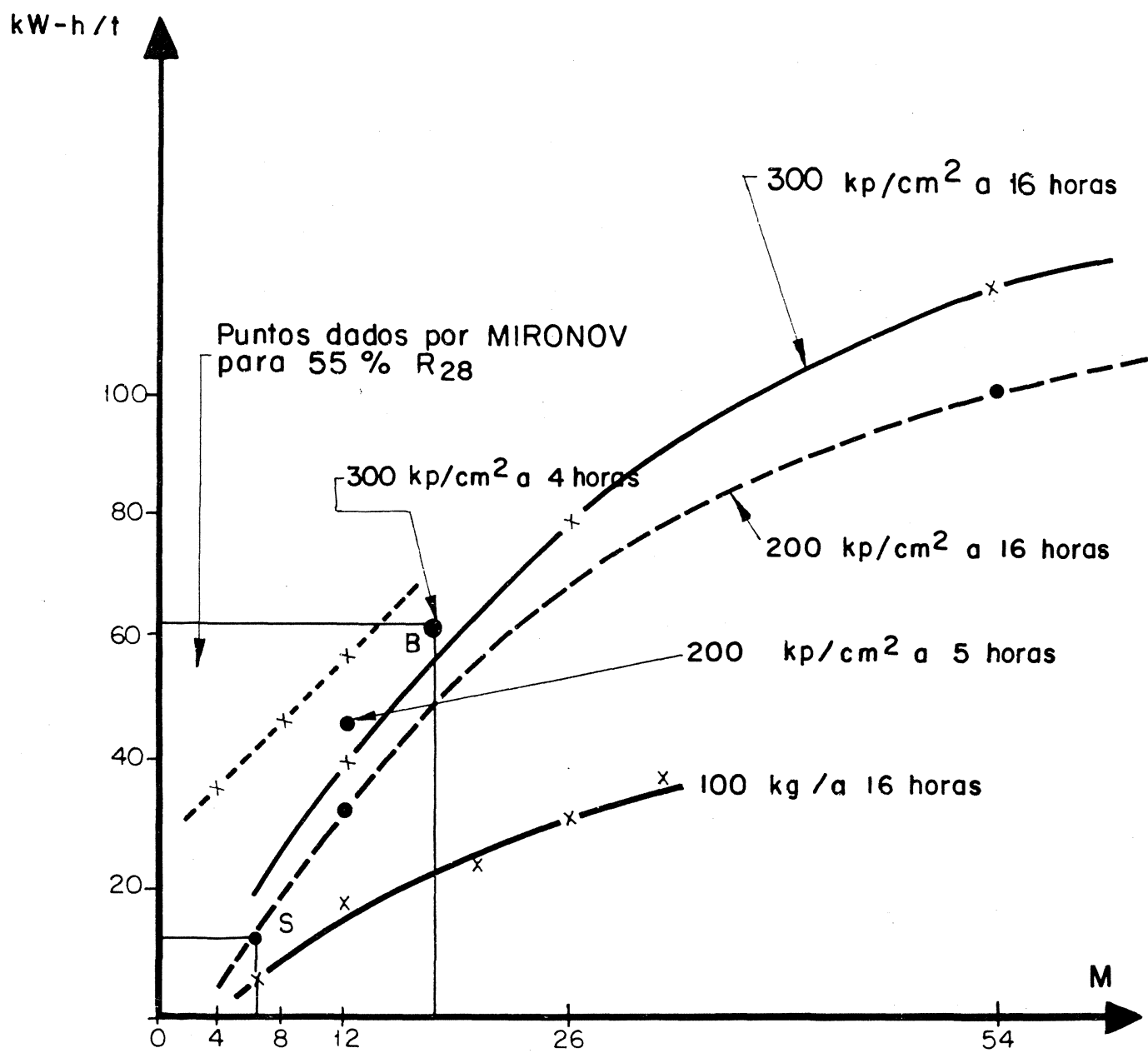

Las $x$ son el resultado de estudios sistemáticos de laboratorio $y$ los son el resultado de experiencias en obra, según C. Martinet.

Fig. 1.-Las horas indicadas se entienden a partir del colado.

Se calcula el factor de madurez (f) mediante la fórmula:

$$
f=(\theta+10) E ;
$$

en la que:

$$
\begin{aligned}
\theta & =\text { temperatura de calefacción. } \\
\mathrm{E} & =\text { edad (en días). } \\
\mathrm{f} & =\text { factor de madurez (en grados } \times \text { día) } .
\end{aligned}
$$


Conociendo el factor (f) se puede entrar en la curva de la figura 3 de Nurse-Saul, obteniendo la resistencia relativa del hormigón. Según indica el profesor Martinet, en la realidad se consiguen hoy en día valores de la resistencia mejores que los obtenidos con la curva de Nurse-Saul, debido posiblemente a que en la actualidad se llega a una mayor finura en el molturado que cuando el profesor Nurse estableció su curva. Propone, por esta razón Martinet, esta otra fórmula para el factor (f):

$$
\begin{aligned}
\mathrm{f} & =(\theta-5) \mathrm{H} . \\
\mathrm{H} & =\text { edad (en horas). }
\end{aligned}
$$

Con la curva necesaria puede asegurarse que los resultados obtenidos actualmente en la práctica corresponden más favorablemente a esta fórmula que a la primitiva.

La fórmula de Rastrup se refiere a la velocidad de las reacciones químicas en función de la temperatura:

donde:

$$
\mathrm{T}_{a}=2 \cdot \frac{\theta-\theta_{a}}{10} \cdot \mathrm{T}
$$

$$
\begin{aligned}
& \mathrm{T}_{a}=\text { tiempo de conservación de la masa a la temperatura } \theta_{a} . \\
& \mathrm{T}=\text { tiempo de conservación de la masa a la temperatura } \theta>\theta_{a} .
\end{aligned}
$$

Lo cual supone que si calentamos a $50^{\circ} \mathrm{C}$ la pasta, cuando el ambiente está a $10^{\circ} \mathrm{C}$, el tiempo de fraguado se reduce en un octavo.

Se ha llegado al convencimiento de que resulta más efectiva una aportación de energía inicial elevada que repartirla en el tiempo con una densidad de energía horaria menor. Es fácil de comprender este extremo considerando, como veremos después, el régimen transitorio de temperaturas en la losa y su relación con el calor aportado.

Las curvas de las figuras 2 y 4 corresponden a ensayos llevados a cabo en laboratorio sobre probetas de $14,1 \times 14,1 \times 56 \mathrm{~cm}$.

Vamos a estudiar en primer lugar el problema térmico suponiendo que el generador de calor se halla sumergido en la masa a calentar: este es el caso concreto de calefacción eléctrica con hilos embutidos en la pasta. Pueden presentarse aquí dos casos diferentes: $\mathrm{A}_{1}$ ) en primer lugar podemos suponer una aportación de calor tan rápida que puede aplicarse un comportamiento adiabático; $\mathrm{A}_{2}$ ) en segundo lugar supondremos que además de calentar la masa se producirán unas pérdidas:

\section{$\mathrm{A}_{1}$ ) Comportamiento adiabático}

La aportación de calor será tal que incrementará la temperatura de la pasta:

$$
\Delta \theta=\frac{\mathrm{Q} \cdot \mathrm{t}}{\mathrm{C}}
$$

siendo:

$$
\begin{aligned}
\mathrm{Q} & =\text { cantidad de calor (en } \mathrm{kcal} / \mathrm{h} \cdot \mathrm{kg}) \\
\mathrm{t} & =\text { tiempo (en horas). } \\
\mathrm{C} & \left.=\text { calor específico (en } \mathrm{kcal} / \mathrm{kg} \cdot{ }^{\circ} \mathrm{C}\right) . \\
\Delta \theta & =\text { incremento de temperatura }\left(\mathrm{en}{ }^{\circ} \mathrm{C}\right) .
\end{aligned}
$$


TEMPERATURAS Y RESISTENCIAS

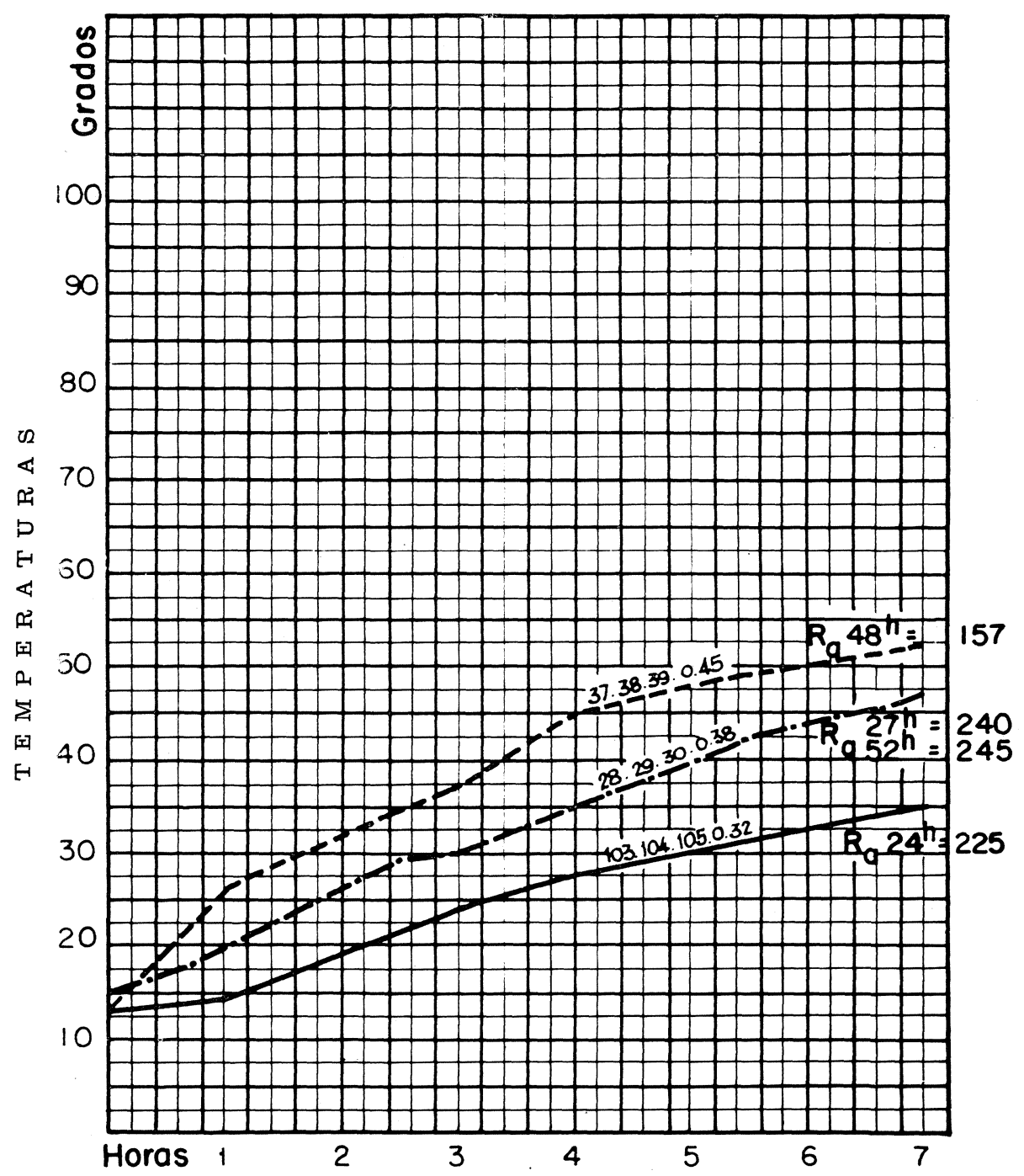

Para $10 \mathrm{kVA} / \mathrm{m}^{3}$ bajo manta aislante (sonda a $15 \mathrm{~mm}$ del límite superior) según C. Martinet.

Fig. 2

\section{$\mathrm{A}_{2}$ ) Calefacción simultánea con pérdidas}

En la realidad el comportamiento de la masa de hormigón no será adiabático, ya que simultáneamente con la calefacción de la masa se producirán pérdidas. Tendremos entonces:

$$
Q \cdot d t=U\left(\theta-\theta_{1}\right) d t+C \cdot d \theta
$$

en la cual:

$$
\mathrm{U}=\text { coeficiente global de pérdidas }\left(\mathrm{en} \mathrm{kcal} / \mathrm{h} \cdot{ }^{\circ} \mathrm{C} \cdot \mathrm{kg}\right) \text {. }
$$




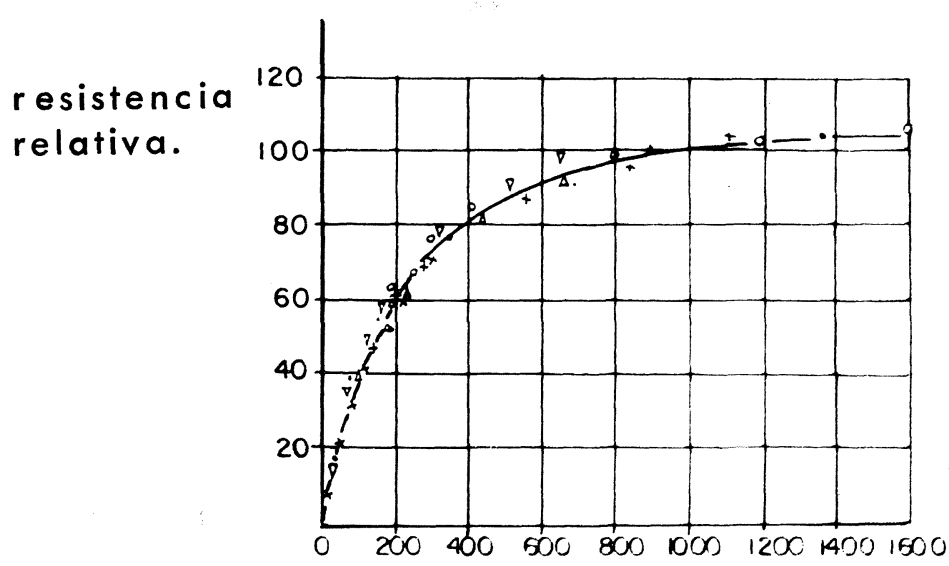

ENSAYOS

- $\quad 46^{\circ} \mathrm{C}$.

- $38^{\circ} \mathrm{C}$

$+29,5$

121

$\nabla \quad 13$

13

4,5

Factor de madurez $=\left(\theta+10^{\circ}\right) \times$ Edad (días)

Fig. 3

Y por tanto:

$$
\Delta \theta=\frac{\mathrm{Q}}{\mathrm{U}}=\left(1-\mathrm{e}^{-\mathrm{t} / \mathrm{c} / \mathrm{u}}\right) .
$$

El coeficiente c/u tiene la dimensión de tiempo; representa este coeficiente el tiempo requerido por la masa hasta alcanzar su temperatura de régimen deseada. Podríamos poner entonces:

$$
\mathrm{T}=\mathrm{c} / \mathrm{u} \text {. }
$$

$\mathrm{Y}$ por tanto:

$$
\Delta \theta=\frac{\mathrm{Q}}{\mathrm{U}}\left(1-\mathrm{e}^{-\mathrm{t} / \mathrm{T}}\right) .
$$

Esta fórmula nos determinará el calor (Q) que es necesario aportar a la masa de hormigón para alcanzar un salto de temperaturas $(\Delta \theta)$ en un tiempo $(\mathrm{t})$. Una vez alcanzado el régimen permanente será necesario continuar aportando una cantidad de calor $\left(Q_{2}\right)$ para anular las pérdidas que se producen.

Se llega al convencimiento así, de que es preferible realizar una aportación inicial de calor superior a la que posteriormente es necesario mantener para conseguir un régimen de temperaturas permanente. Este extremo está de acuerdo con los resultados experimentales citados anteriormente.

Podemos calcular el tiempo inicial $\left(\mathrm{T}_{1}\right)$ en el que la aportación de calor ( $\left.\mathrm{Q}^{\prime}\right)$ sea muy superior a la de régimen normal $\left(Q_{\theta},=Q^{\prime}>Q_{n}\right)$ sin sobrepasar una temperatura $\left(\theta^{\prime}\right)$ que podamos considerar como límite de seguridad. Tomando logaritmos tendremos:

$$
\mathrm{T}_{1}=\mathrm{T} \cdot \ln \cdot \frac{\mathrm{Q}^{\prime}}{\frac{\mathrm{Q}_{\mathrm{n}}}{\mathrm{K}-1}}
$$

El ingeniero C. Martinet ha llegado a la fórmula:

$$
\theta=\frac{3 \mathrm{P}}{\lambda}\left(1-\mathrm{e}^{-\mathrm{t} / 6 \mathrm{~T}}\right) \text {. }
$$




\section{CURVAS DE TEMPERATURA}

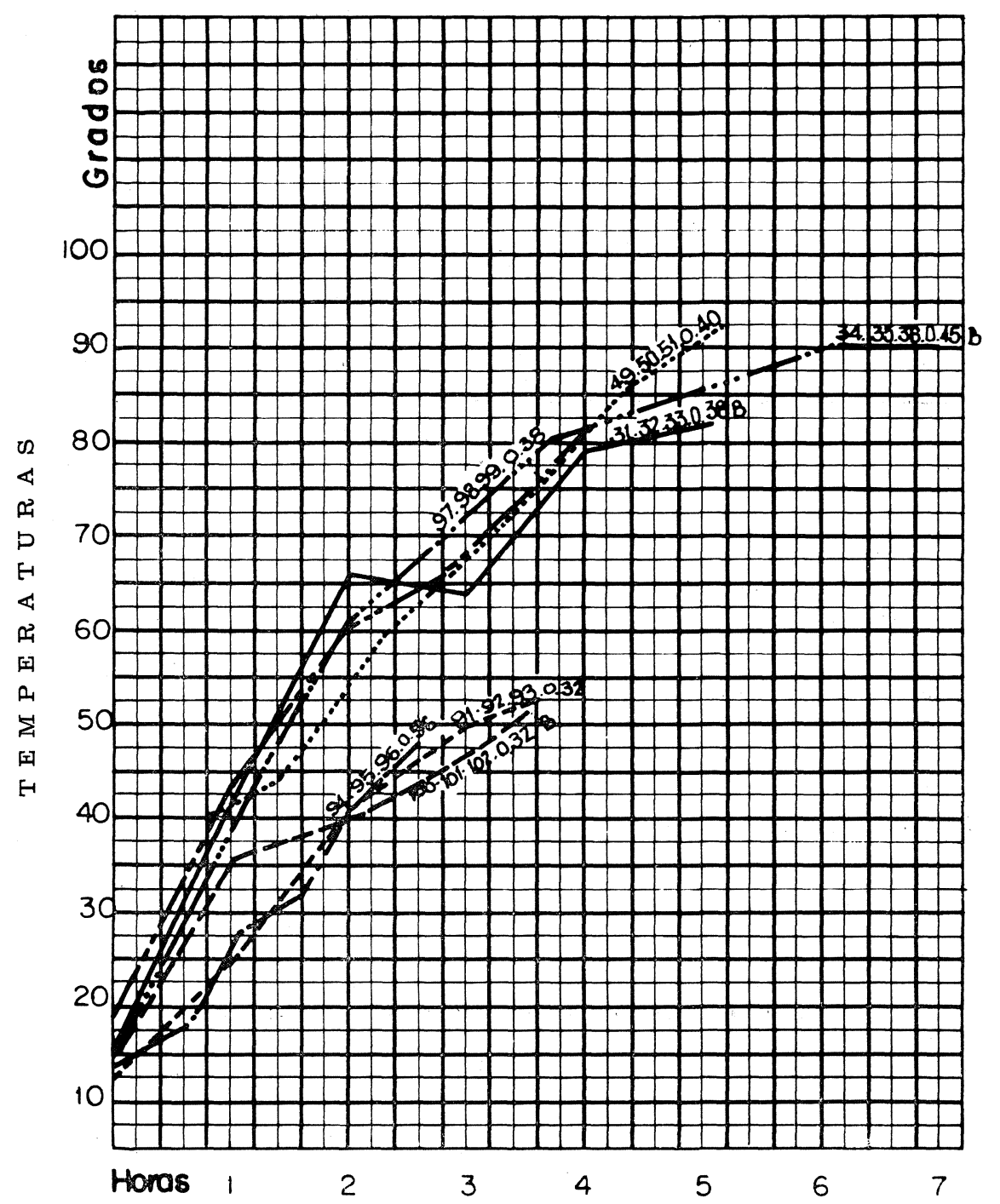

Para $20 \mathrm{kVA} / \mathrm{m}^{3}$ (sonda a $15 \mathrm{~mm}$ del límite superior) según C. Martinet.

Fig. 4

Esta fórmula está representada en la figura 5, en donde $\mathrm{P}$ equivale a la aportación de calor (en $\mathrm{W})$ y $\lambda$ al coeficiente de transmisión térmica global (en $\mathrm{W} /{ }^{\circ} \mathrm{C}$ ).

\section{Distribución de temperaturas}

La losa de hormigón que forma el forjado puede encontrarse en distintas hipótesis de tratamiento térmico:

$\mathrm{B}_{1}$ ) Calefacción en la cara inferior, sin aislamiento en la exterior.

$\mathrm{B}_{2}$ ) Calefacción en la cara inferior, con aislamiento en la exterior.

$\mathrm{B}_{3}$ ) Calefacción en ambas caras. 


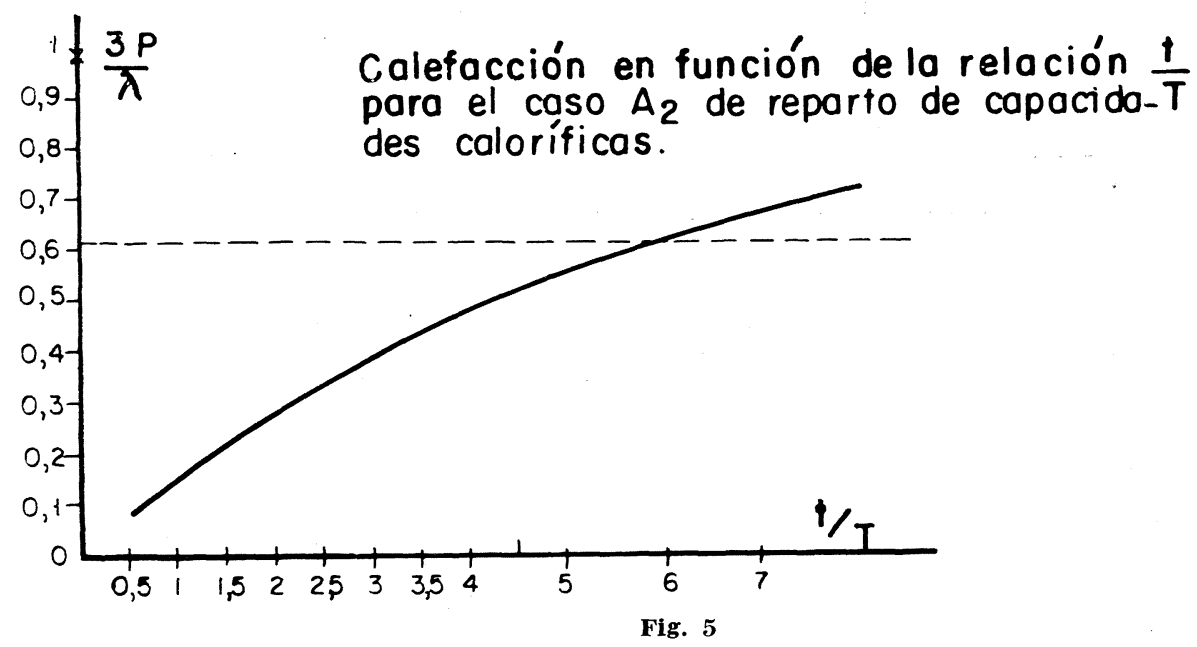

En cada caso tendremos una distribución de temperaturas diferente, así como unas pérdidas térmicas también distintas. Vamos a estudiar cada caso por separado:

$\left.\mathrm{B}_{1}\right)$ Calefacción en la cara inferior, sin aislamiento en la exterior

Suponiendo el caso más sencillo de que se hayan alcanzado condiciones estacionarias, la distribución de temperaturas será la indicada en la figura 6. Se advierte cómo la capa superficial de hormigón estará a una temperatura próxima a la del ambiente exterior. (Posiblemente esté a una temperatura inferior al tener en cuenta la pérdida de agua por evaporación que supone una refrigeración para esta capa).

DISTRIBUCION DE TEMPERATURAS

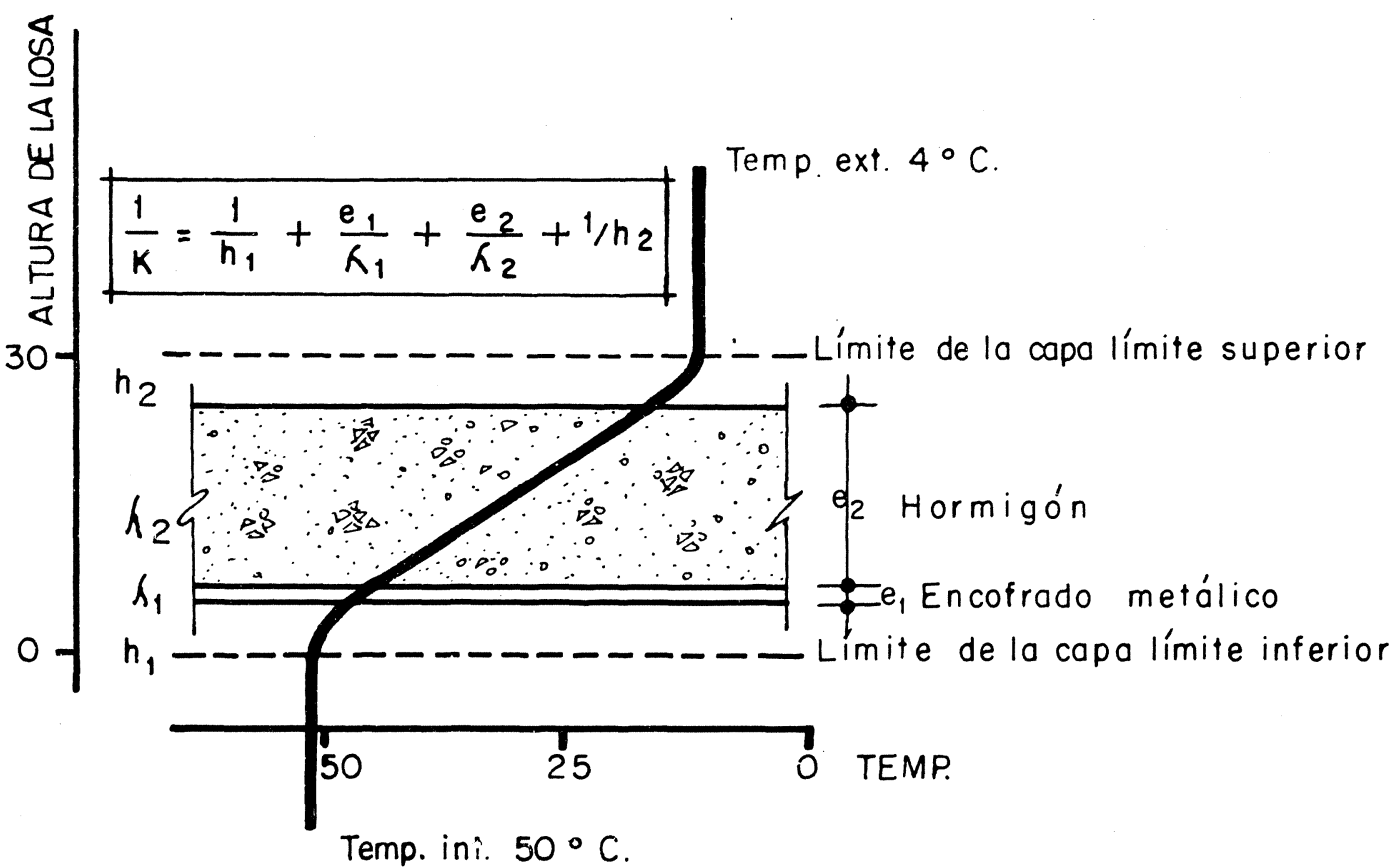

Fig. 6.-Distribución vertical de temperaturas en el caso $B_{1}$, régimen estacionario. 
En el caso más general deberemos suponer un régimen transitorio, que necesariamente ocurre durante el tiempo que la losa tarda en calentarse. En estas condiciones la distribución de temperaturas vendrá determinada por la fórmula:

en la cual:

$$
\nabla^{2} \theta=\frac{1}{\mathrm{~h}^{2}} \cdot \frac{\partial \theta}{\partial \mathrm{t}}
$$

$$
\nabla^{2}=\text { operador laplaciana. }
$$

Resolviendo esta ecuación para las condiciones límites de nuestro caso llegaríamos a la fórmula :

$$
\theta(t)=\frac{\mathrm{Q} \cdot \mathrm{d}}{\mathrm{K}}\left[1-\frac{8}{\pi^{2}} \sum_{0}^{\infty}\left(2_{\mathrm{n}}+1\right)^{-2} \mathrm{e}^{-2\left(2_{\mathrm{n}}+1\right)} \cdot \pi^{2} \cdot \mathrm{K} \cdot \mathrm{t} / 4 \mathrm{~d}^{2} \cdot \mathrm{C}\right]
$$

donde:

$\theta(t)=$ distribución de temperatura según el tiempo en la cara latente.

$\mathrm{Q}=$ flujo de calor (en $\mathrm{kcal} / \mathrm{m}^{2} \cdot \mathrm{h}$ ).

$\mathrm{d}=$ espesor del forjado.

$\mathrm{K}=$ coeficiente de conductibilidad.

$\mathrm{t}=$ tiempo.

$\mathrm{C}=$ capacidad calorífica por unidad de volumen (densidad $\times$ calor específico).

La representación gráfica de la ecuación anterior coincide con la de la figura 5 .

Si deseamos conocer la distribución de temperaturas en régimen permanente en toda la sección del forjado, deberemos resolver la ecuación:

$$
\frac{\partial^{2} \mathrm{t}}{\partial \mathrm{x}^{2}}+\frac{\partial^{2} \mathrm{t}}{\theta_{\mathrm{y}} \cdot \partial \mathrm{y}^{2}}=0
$$

Para las condiciones límites de nuestro caso: un plano a temperatura constante $[t=$ $\left.=\mathrm{T}\left(50^{\circ} \mathrm{C}\right)\right] \mathrm{y}$ el plano superior y los paramentos laterales a temperatura constante diferente, que podemos suponer $0^{\circ} \mathrm{C}$. En este caso la distribución de temperaturas en el interior de la losa estará determinada por la fórmula:

$$
\frac{\mathrm{t}}{\mathrm{T}}=2 \sum_{1}^{\infty} \frac{\mathrm{n} \cdot \pi}{\left[1-(-1)^{\mathrm{n}}\right]}\left(\frac{\mathrm{Sh} \frac{\mathrm{n} \cdot \pi \cdot \mathrm{v}}{\mathrm{L}}}{\mathrm{Sh} \frac{\mathrm{n} \cdot \pi \cdot \mathrm{W}^{\prime}}{\mathrm{L}}}\right) \cdot \operatorname{sen} \frac{\mathrm{n} \cdot \pi \cdot \mathrm{x}}{\mathrm{L}} .
$$

(Su representación gráfica está dada en la figura 7). Se ha corregido ligeramente la distribución en los laterales teniendo en cuenta el pequeño aislamiento que supone el encofrado en esos puntos.

La figura 6 supone un corte de la figura 7 por un plano perpendicular.

En resumen, podemos advertir cómo en el caso de calefacción de la cara inferior del forjado, sin ningún aislamiento en la exterior, se origina un marcado gradiente de temperaturas, que aumentará si se superpone el fenómeno de evaporación que necesariamente se produce si se deja en contacto libre con la atmósfera el plano superior de la losa; simultáneamente se producirá un incremento de las pérdidas caloríficas fácilmente evaluables. 


\section{$\mathrm{B}_{2}$ ) Calefacción en la cara inferior, con aislamiento en la exterior}

Si suponemos que aislamos la cara superior de la losa, podremos mantener en la misma una temperatura superior que la exterior. Esta temperatura será función del poder aislante del material utilizado; esto es, de su conductibilidad $(\lambda)$ y de su espesor (e).

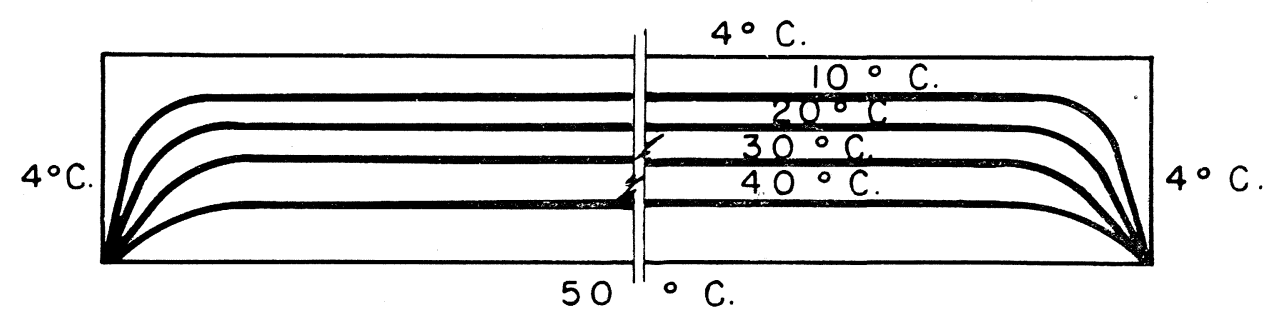

Fig. 7.-Distribución de temperaturas en una sección, caso $B_{1}$. Régimen estacionario.

Podríamos calcular esta temperatura $\left(\mathrm{T}_{a}\right)$ gracias al sistema de ecuaciones:

$$
\begin{aligned}
& \mathrm{Q}_{2}=\mathrm{K}_{\mathrm{t}}\left(\mathrm{T}_{\mathrm{i}}-\mathrm{T}_{\mathrm{e}}\right) \\
& \mathrm{Q}_{2}=\left(\frac{1}{\frac{1}{\mathrm{~h}_{\mathrm{i}}}+\frac{\mathrm{e} \cdot \mathrm{h}}{\lambda_{\mathrm{h}}}}\right) \cdot\left(\mathrm{T}_{\mathrm{i}}-\mathrm{T}_{a}\right)=\left(\frac{1}{\frac{1}{\mathrm{~h}_{2}}+\frac{\mathrm{e}_{a}}{\lambda_{a}}}\right) \cdot\left(\mathrm{T}_{a}-\mathrm{T}_{\mathrm{e}}\right) \\
& \frac{1}{\mathrm{~K}_{\mathrm{t}}}=\frac{1}{\mathrm{~h}_{\mathrm{i}}}+\frac{\mathrm{e} \cdot \mathrm{h}}{\lambda_{\mathrm{h}}}+\frac{1}{\mathrm{~h}_{2}}+\frac{\mathrm{e}_{a}}{\lambda_{a}} .
\end{aligned}
$$

La utilización de una capa aislante supone un ahorro de energía que puede calcularse a partir del sistema:

$$
\begin{aligned}
\text { Ahorro } & =\mathrm{Q}_{1}-\mathrm{Q}_{2} ; \\
\mathrm{Q}_{1} & =\mathrm{K}_{1} \cdot \Delta \mathrm{T} ; \\
\mathrm{Q}_{2} & =\mathrm{K}_{2} \cdot \Delta \mathrm{T} ; \\
\frac{1}{\mathrm{~K}_{2}} & =\frac{1}{\mathrm{~K}_{1}}+\frac{\mathrm{e}_{a}}{\lambda_{a}} ;
\end{aligned}
$$

donde:

$$
\begin{aligned}
\Delta \mathrm{T} & =\text { temperatura diferencial interior, exterior. } \\
\mathrm{K}_{1} & =\text { coeficiente transmisión aire-aire sin aislamiento. } \\
\mathrm{e}_{a} & =\text { espesor del aislamiento. } \\
\lambda_{a} & =\text { coeficiente de conductibilidad del aislamiento. }
\end{aligned}
$$

Esta instalación de aislamiento supone en la obra unos gastos adicionales, no sólo de amortización de la inversión, sino también de colocación y levantamiento diario, grúa, rhano de obra, espacio ocupado durante el colado, etc... Por tanto, aunque desde el punto de vista técnico está totalmente justificado el aislamiento, ya que evitará la evaporación del agua (si es impermeable esa capa) y aminorará el gradiente de distribución de 
temperaturas en el seno del forjado, por lo que éste fraguará en su conjunto simultáneamente, y no sucesivamente por capas, como parece que ocurre en el fraguado sin aislamiento superior.

El ahorro de energía (en \%) será:

$$
\text { Ahorro de energía }(\%)=\frac{\mathrm{Q}_{1}-\mathrm{Q}_{2}}{\mathrm{Q}_{1}}=1-\frac{1}{1+\frac{\mathrm{e}_{a}}{\lambda_{a}} \cdot \mathrm{K}_{1}}
$$

Teniendo en cuenta que el valor de $\mathrm{K}_{1}$ para el hormigón es $\approx 1,3$ y que $\lambda_{a}=0,032$ tendremos la siguiente tabla:

$\begin{array}{cc}\text { Espesor del aislamiento } & \text { Ahorro de energia (\%) } \\ 1 \mathrm{~cm} & 28 \\ 2 \mathrm{~cm} & 44 \\ 3 \mathrm{~cm} & 54 \\ 4 \mathrm{~cm} & 60\end{array}$

Puede advertirse que este problema debe centrarse en un clásico ejemplo de minimización de costos. En el caso general deberá tenerse en cuenta la amortización de los costes de primera instalación: coste del aislamiento montado, y el costo de explotación que genera este aislamiento, ya que deberá ponerse y quitarse una vez en cada ciclo. En contraposición tendremos una disminución de la energía consumida, determinada por la tabla anterior.

En la figura 8 se representa este ejemplo sin determinar parámetros, puesto que desconocemos los costos de explotación que generan este aislamiento. Puede advertirse cómo el coste de amortización del aislamiento imputado sobre cada forjado construido, bajo la hipótesis de que se utiliza 300 veces en la obra, es mínimo: aproximadamente 0,2 ptas/ $/ \mathrm{m}^{2} \cdot \mathrm{cm}$ de espesor. El problema de utilizar o no el aislamiento viene determinado por los costes directos de grúa y mano de obra que resultan de su movimiento diario.

No es necesario recordar otra vez que, además del problema de pérdida de energía por transmisión visto, hay que considerar las pérdidas por evaporación que se eliminarían haciendo impermeable el aislante, lo cual no supone ningún coste excesivo.

\section{$\mathrm{B}_{3}$ ) Calefacción en ambas caras}

En este caso el reparto de temperaturas en la losa será uniforme. Desde un punto de vista práctico no parece necesario este sistema, salvo en el caso de que la temperatura exterior fuera tan baja, por debajo de $-10^{\circ} \mathrm{C}$, que un aislamiento normal no impidiera temperaturas de escarcha en la capa superficial del hormigón.

Unicamente en zonas de alta montaña pueden concurrir todas estas causas. En estos casos una manta eléctrica calefactora y aislante, posiblemente sea la mejor solución.

Otro posible caso de calefacción por ambas caras se presenta cuando calentamos la inferior por medios convencionales y simultáneamente tratamos con vapor la cara superior, para así anular también las pérdidas de agua. Este sistema, indudablemente más sofisti- 
cado, sólo será rentable si la cara superior se ha aislado del ambiente con una capa o lona, impermeable, que cree una atmósfera saturada sobre el hormigón. Al referirnos a las pérdidas por evaporación volveremos sobre este tema.

\section{COSTES SEGUN ESPESOR AISLAMIENTO}

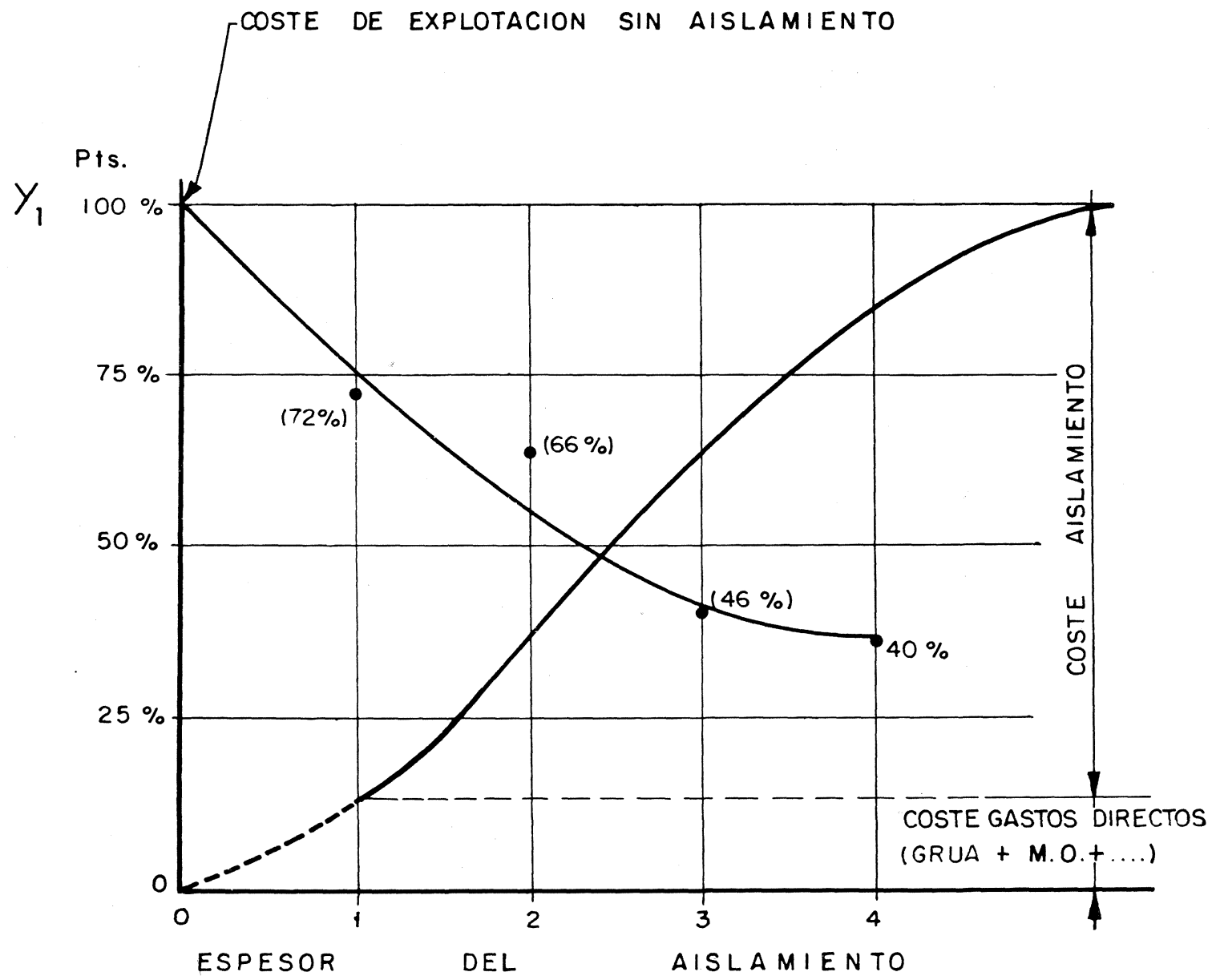

(El punto $\mathrm{y}_{1}$ resulta $8 \mathrm{ptas} / \mathrm{m}^{2}$ para un costo del combustible gas-oil de 2,33 ptas/litro).

Nota: El coste del panel aislante montado oscila sobre unas $60 \mathrm{ptas} / \mathrm{m}^{2}$ para $1 \mathrm{~cm}$ de espesor. Suponiendo que cada panel se amortice en un año, esto es, en 300 noches laborables tendríamos una imputación por amortización de aislamiento definida par la presente tabla.

\begin{tabular}{|c|c|}
\hline ESPESOR & COSTE IMPUTADO \\
\hline 0 & 0 \\
1 & $0,2 \mathrm{ptas} / \mathrm{m}^{2}$ \\
2 & $0,4 \mathrm{ptas} / \mathrm{m}^{2}$ \\
3 & $0,57 \mathrm{ptas} / \mathrm{m}^{2}$ \\
4 & $0,7 \mathrm{ptas} / \mathrm{m}^{2}$ \\
\hline
\end{tabular}

Fig. 8.-Cálculo del espesor de aislamiento mínimo. 


\section{Pérdidas de calor latente}

Entre la pasta de hormigón y el ambiente exterior se producirá un transporte de agua en forma de vapor, que requerirá una aportación de calor para permitir el cambio de estado. Esta energía habrá sido tomada de la propia pasta: el hormigón pierde agua y pierde energía.

La cantidad de vapor de agua (W') perdida por la pasta vendrá determinada por la diferencia entre la humedad relativa del aire y su presión parcial correspondiente, y la presión de vapor a la temperatura a que se encuentra la pasta. Indudablemente el factor aire o viento tiene una gran importancia, ya que si el día es calmado se podrá crear una capa límite saturada en contacto con la pasta que frene esta evaporación; en estas condiciones, la fórmula siguiente nos expresará las pérdidas de agua $\mathrm{W}^{\prime}\left(\mathrm{en} \mathrm{g} / \mathrm{h} \cdot \mathrm{m}^{2}\right)$ :

$$
\mathrm{W}^{\prime}=9\left(\mathrm{~T}-\mathrm{T}^{\prime}\right) \text {. }
$$

Si existe una corriente de aire paralela a la superficie tendremos:

$$
\mathrm{W}^{\prime}=9\left(1+\frac{\mathrm{V}}{1,16}\right)\left(\mathrm{T}-\mathrm{T}^{\prime}\right) \text {. }
$$

Y si la corriente de aire es vertical:

$$
\mathrm{W}^{\prime}=18\left(1+\frac{\mathrm{V}}{1,27}\right)\left(\mathrm{T}-\mathrm{T}^{\prime}\right) .
$$

Siendo:

$$
\begin{aligned}
\mathrm{V} & =\text { velocidad del aire }(\mathrm{en} \mathrm{m} / \mathrm{s}) \\
\mathrm{T} & =\text { temperatura seca del aire }\left(\mathrm{en}{ }^{\circ} \mathrm{C}\right) \\
\mathrm{T}^{\prime} & =\text { temperatura húmeda del aire }\left(\mathrm{en}{ }^{\circ} \mathrm{C}\right) . \\
\mathrm{W}^{\prime} & =\text { evaporación de agua }\left(\mathrm{en} \mathrm{g} / \mathrm{h} \cdot \mathrm{m}^{2}\right) .
\end{aligned}
$$

La pérdida de calor que corresponde a estas pérdidas de masa vendrá definida por la expresión:

$$
\mathrm{Q}_{l}=\mathrm{W}^{\prime} \cdot 0,6 \mathrm{kcal} / \mathrm{h} \cdot \mathrm{m}^{2} .
$$

Pero además se producirá una pérdida de energía sensible en el hormigón dada por la fórmula:

$$
\mathrm{Q}_{\mathrm{s}}=\left(\mathrm{T}-\mathrm{T}_{a}\right) \mathrm{W}^{\prime}\left(\mathrm{en} \mathrm{kcal} / \mathrm{h} \cdot \mathrm{m}^{2}\right) \text {; }
$$

en la cual:

$$
\begin{aligned}
& \mathrm{T}=\text { temperatura de la pasta. } \\
& \mathrm{T}_{a}=\text { temperatura del agua aportada para restituir la evaporación. }
\end{aligned}
$$

Desgraciadamente es difícil de determinar esta pérdida $Q_{2}$, ya que al realizarse la aportación de agua, mediante riego superficial, ésta no se dosifica ni controla y, por tanto, las pérdidas son superiores a las necesarias.

Realmente, durante la noche, las temperaturas húmedas difieren poco de las temperaturas secas, y menos aún en zonas marítimas; en la figura 9 se presenta un clımatograma tipo en el que puede advertirse cómo en un día de verano, con temperaturas máximas de $32^{\circ} \mathrm{C}$, por la noche la diferencia $\mathrm{T}-\mathrm{T}$ ' es muy pequeña. Si la humedad relativa es del $100 \%$ no se producirán pérdidas por evaporación (salvo corrientes de aire elevadas). 
El control de la humedad de la pasta puede no ser un problema que haya que resolver por otro medio que el simple riego en climas húmedos. En climas secos continentales, como los de la meseta castellana, por el contrario será necesario un enfoque particular del problema, existiendo varias soluciones:

1. ${ }^{\circ}$ Riego superficial: Pérdidas térmicas excesivas.

2. ${ }^{\circ}$ Cubrir la pasta con lona impermeable: Se crea una capa saturada que minimiza las pérdidas.

3.') Cubrir la pasta con lona o manta impermeable y tratamiento de vapor: Su costo elevado sólo se justifica con temperaturas exteriores muy bajas.

VARIACION DE LA TEMPERATURA

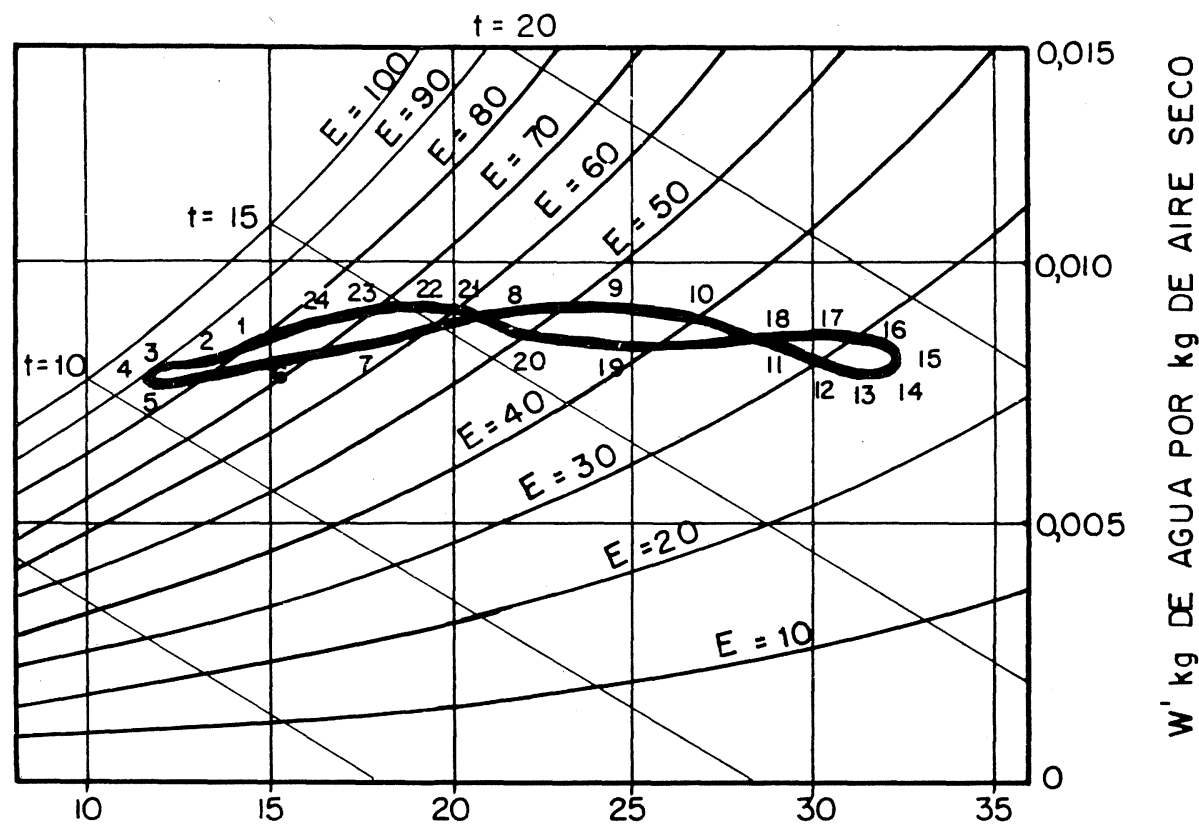

T E M P E A T U R A S

(Las cifras alrededor del climatograma indican las horas)

C L I M A T O G R A A

Fig. 9.-Variación de la temperatura y la humedad del aire exterior a lo largo del dia.

C. Martinet, en el Congreso F.I.P. de Praga (junio 1970) indicó que, para temperaturas de $30^{\circ}$ a $40^{\circ} \mathrm{C}$, los grados hora necesarios para obtener el $65 \%$ de la resistencia a los 28 días se sitúa entre 1.200 y 2.000 , lo cual supone por $t=40^{\circ} \mathrm{C}$ un número de 30 a 50 horas. En este mismo informe el autor propugna por un sistema de manta impermeable e inyección de vapor a $60^{\circ} \mathrm{C}$; en estas condiciones se reducen para una viga de $0,14 \mathrm{~m}$ de espesor 
las pérdidas de agua, en 7 días, a $2 \mathrm{kp} / \mathrm{m}^{2}$ mientras que en condiciones normales sería de $5 \mathrm{kp} / \mathrm{m}^{2}$ y con endurecimiento eléctrico, de $10 \mathrm{~kW} / \mathrm{m}^{3}$, en 8 horas de calefacción se alcanzarían los $6 \mathrm{kp} / \mathrm{m}^{2}$. Para las primeras 24 horas tendríamos los siguientes valores:

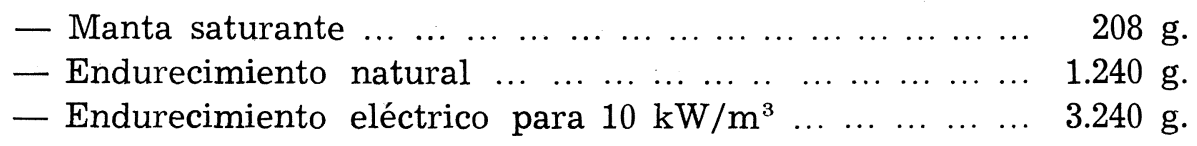

Es necesario destacar por último que el riego normal no sólo supone una pérdida de energía, sino que también perjudica la distribución de temperaturas en el seno de la pasta ya que el agua normalmente estará a una temperatura inferior al ambiente (salvo días de mínima extremada). Y no debe olvidarse que el tratamiento térmico del hormigón no se requiere por la existencia de reacciones endotérmicas de fraguado (muy al contrario se genera calor) si no a que estas reacciones, exotérmicas, se aceleran al elevarse la temperatura, por lo que un gradiente elevado en la losa supondrá una velocidad de fraguado diferente según cada capa, y posiblemente un hormigón menos homogéneo.

\section{Resumen}

Llegamos, al final de nuestro estudio teórico, a las siguientes conclusiones:

1. $\left.{ }^{\circ}\right)$ Las reacciones de fraguado son exotérmicas.

2..$^{\circ}$ Estas reacciones se aceleran al elevar la temperatura de la pasta.

3..$^{\circ}$ En el cálculo de potencia sólo deberán tenerse en cuenta los siguientes factores:

- pérdidas por transmisión y convención;

- pérdidas de calor latente, y

- aportación para incrementar la temperatura de la pasta.

4. ${ }^{\circ}$ Se recomienda una aportacion de energía en principio tan grande que permita una rápida elevación de la temperatura del hormigón.

5..$^{\circ}$ Parece aconsejable un gradiente vertical de temperaturas homogéneo en la losa.

6. ${ }^{\circ}$ Esta distribución de temperaturas deseada se consigue calentando una cara y aislando la contraria.

7. ${ }^{\circ}$ Si económicamente no es aconsejable utilizar un panel aislante en la capa superior, técnicamente parece obligado el calentamiento de esta capa superior por otros medios (resistencias eléctricas sumergidas, vapor, etc.) o utilizar algún sistema que acelere el endurecimiento de esta capa (aditivos, etc.).

\section{COSTOS Y CARACTERISTICAS DE LOS DISTINTOS SISTEMAS}

A continuación vamos a referirnos al costo de la termia obtenida a través de distintos combustibles en la España peninsular, para a continuación referirnos a los distintos sistemas de calefacción que pueden utilizarse.

\section{COSTe de la energia en ha Peninsula}

En la tabla I presentamos un cuadro del coste de la kcal/h, en función del combustible utilizado. Las columnas de la derecha se refieren a los costos reales en función del rendimiento que pueda conseguirse en cada caso. 
T A B L A I

Comparación de precios de diversos combustibles

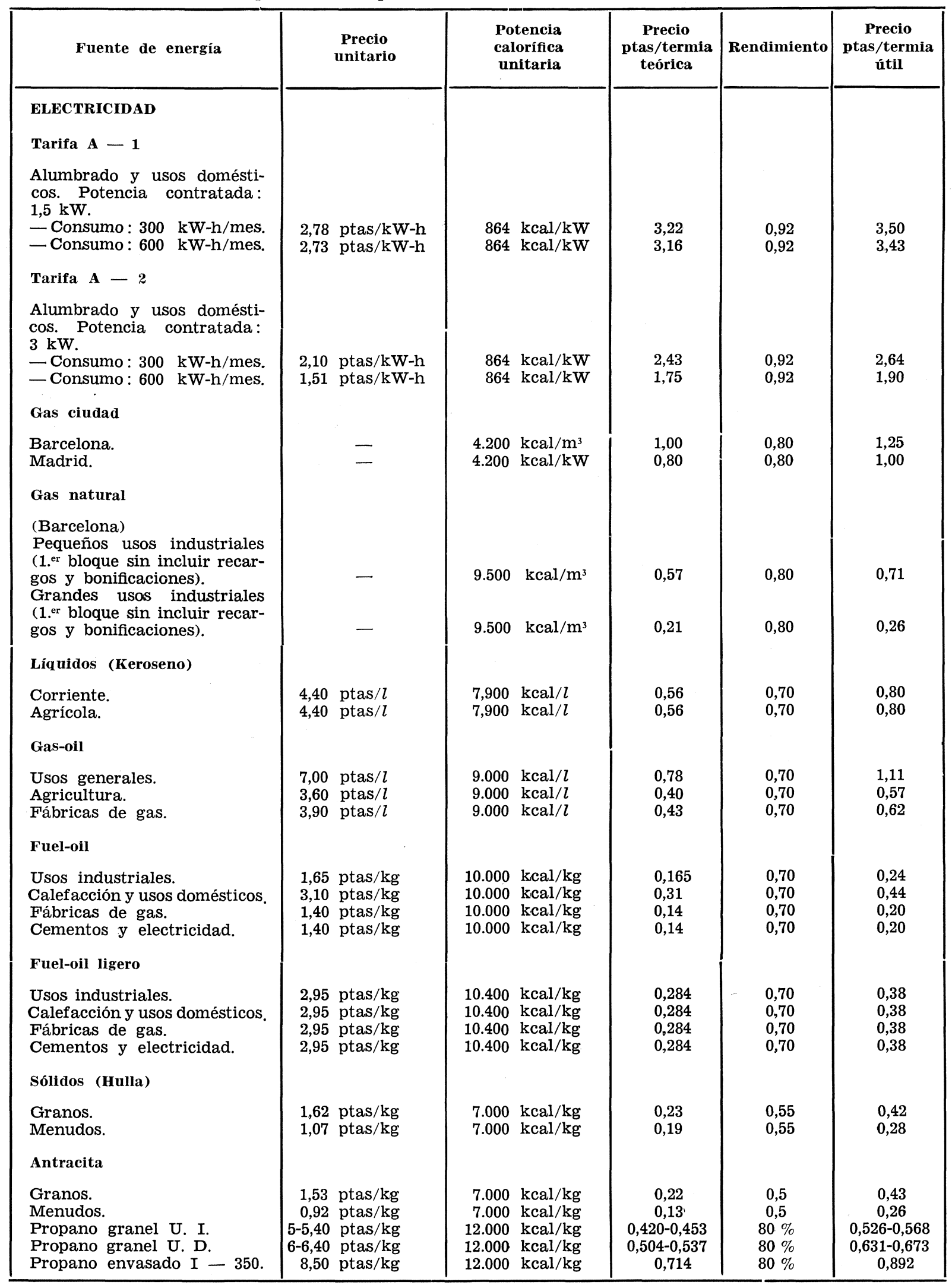


En un rápido estudio puede advertirse cómo el combustible más económico resulta el fuel-oil industrial, pero su utilización presenta grandes inconvenientes debido a su excesiva densidad. Por esta razón, y descontando el carbón y la leña, parece que los combustibles más económicos son el petróleo y el gas propano. Así como el petróleo comercial es un combustible fácil de encontrar en cualquier lugar, el propano tiene un suministro más restringido, además de que obliga a cumplir una legislación especial sobre combustibles en depósitos a presión.

La electricidad, a pesar de su gran utilización en otros países con este fin, no presenta ventajas económicas en nuestra patria. En Francia el constructor puede beneficiarse por la noche de tarifas de valle, con un coste aproximado de $0,04 \mathrm{fr} / \mathrm{kW} \cdot \mathrm{h}$, equivalente a unas $0,50 \mathrm{ptas} / \mathrm{kW} \cdot \mathrm{h}$.

En nuestro país nos encontramos con precios del $\mathrm{kW}$ del orden del doble o más. En las tarifas actuales existen tres bloques de precio descendente, según el número de $\mathrm{kW} \cdot \mathrm{h}$ consumidos. Suponiendo que la potencia instalada en servicios generales de día es semejante a la de iluminación y fraguado de noche, podría llegarse a considerar, de una forma puramente teórica, que los $\mathrm{kW} \cdot \mathrm{h}$ consumidos de noche pertenecen al tercer bloque, y que el $10^{\circ}$ y $2 .^{\circ}$ bloque se cubren con el consumo del día.

La tabla I se ha realizado suponiendo un precio medio o resultante del $\mathrm{kW} \cdot \mathrm{h}$, según las tarifas actuales en la España peninsular.

El decreto 1968/1969 del 16 de agosto, sobre tarifas eléctricas, se refiere a una fórmula binómica $(\mathrm{A}+\mathrm{r})$, en donde: $\mathrm{A}$ es el sumando imputado a la empresa vendedora, compuesto por:

$$
A_{p}+A_{e}
$$

siendo:

$A_{p}=$ término de potencia.

$\mathrm{A}_{\mathrm{e}}=$ primera parte del término de energía.

$\mathrm{r}=$ segunda parte del término de energía, correspondiente a OFILE.

En definitiva la tarifa es del tipo:

Tarifa total $=$ término de potencia $\left(A_{p}\right)+$ término de energía $\left(A_{e}+r\right)$.

Esta nueva reglamentación supone que al aumentar el consumo, para una potencia instalada dada, el coste del $\mathrm{kW} \cdot \mathrm{h}$ disminuye. Por otro lado, presenta la calefacción eléctrica indudables ventajas de tipo práctico, que también deben considerarse:

- mínimo espacio ocupado ;

- mínimo gasto en transporte y acarreos de elementos dentro de la obra;

- fácil control ;

- seguridad de funcionamiento ;

- seguridad de aprovisionamiento;

— seguridad de accidentes utilizando bajos voltajes ;

- limpieza total;

- flexibilidad de funcionamiento, y

- movilidad de los elementos y su distribución en obra. 


\section{DIFERENTES SISTEMAS DE APORTACION TERMICA}

Hemos determinado el abanico de costes de la termia que se pueden presentar al constructor, en función de los posibles diferentes combustibles que pueden utilizarse.

Para tomar una decisión en cuanto al sistema calefactor a adoptar, además de conocer esta información, es necesario ponderar también las diferentes modalidades de aportación térmica que puedan adoptarse.

El calor puede transmitirse hasta la losa de hormigón con ayuda de alguno de estos medios:

- aire;

- agua;

- vapor;

- fluido térmico, o

- directamente.

El aire, a pesar de su pequeño calor específico $0,24 \mathrm{kcal} / \mathrm{kg}$, tiene grandes ventajas, ya que no exige su transporte hasta el generador de calor y permite su libre pérdida si es necesario.

El agua exige tuberías, equipos mecánicos, etc., que no siempre pueden utilizarse en obra. Un fluido térmico, además, presentaría el inconveniente de un elevado costo por unidad de capacidad.

El vapor, desde los puntos de vista psicrométrico y térmico sería el mejor transmisor de energía, pero requiere unidades generadoras de mayor volumen y mayor vigilancia.

\section{CAlCulo de la potencia real requerida $Y$ temperatura DEL HORMIGON}

La experiencia adquirida en otros países referente a las necesidades de potencia calorífica, se centra en los estudios realizados con electricidad; se llegan así a potencias, en forjados de pisos, de 800 a $1.000 \mathrm{~W} / \mathrm{m}^{2}$, durante 8 horas de calentamiento. Supondremos que estas potencias citadas son globales e incluyen:

- calor requerido para calentar la masa hasta $50^{\circ} \mathrm{C}$;

- calor perdido por evaporación del agua en la superficie libre superior y pérdidas por aportación de agua fresca, y

- calor perdido por transmisión a través del hormigón y convención con el aire.

Hemos seleccionado esta temperatura de $50^{\circ} \mathrm{C}$, que puede considerarse como conservadora, ya que existen experiencias hasta para $90^{\circ} \mathrm{C}$. El límite de temperatura estarà dado por la de ebullición del agua a la presión local $\left(100^{\circ} \mathrm{C}\right.$ a $960 \mathrm{~mm}$ de $\left.\mathrm{Hg}\right)$, ya que en ese momento la pérdida de agua sería tan rápida que perturbaría el fraguado de la pasta. Una temperatura de $50^{\circ} \mathrm{C}$ en el hormigón y en el agua en él contenida supone para ésta una presión de vapor de $0,12578 \mathrm{kp} / \mathrm{cm}^{2}$. Al aumentar la temperatura, aumentará su presión de vapor hasta la presión atmosférica local) y, por tanto, también aumentará su diferencia con la presión parcial del vapor de agua del aire, incrementándose la evaporación y, como consecuencia, las pérdidas térmicas según $0,6 \mathrm{kcal} / \mathrm{g}$ de agua evaporada. 


\section{CALOR PERDIDO POR TRANSMISION A TRAVES DEL HORMIGON Y CONVENCION CON EL AIRE}

El coeficiente $(\mathrm{K})$ de nuestro forjado será:

$$
\begin{array}{rlrl}
\frac{1}{\mathrm{~K}} & =\frac{1}{25}+\frac{1}{10}+\frac{0,15}{1,30} ; & & - \text { para } \mathrm{h}_{\mathrm{e}}=25 \mathrm{kcal} / \mathrm{m}^{2} \cdot \mathrm{h} ; \\
\mathrm{K} & =3,9 \mathrm{kcal} / \mathrm{m}^{2} \cdot \mathrm{h} . & & - \text { para } \mathrm{h}_{\mathrm{i}}=10 \mathrm{kcal} / \mathrm{m}^{2} \cdot \mathrm{h} ; \\
& & - \text { para hormigón }=1,30 \mathrm{kcal} / \mathrm{m}^{2} \cdot \mathrm{h} ;
\end{array}
$$

Y por tanto:

$$
\begin{aligned}
\mathrm{Q} & =\Delta \mathrm{t} \cdot \mathrm{K} ; \\
\Delta \mathrm{t} & =46^{\circ} \mathrm{C}(\text { diferencias de temperaturas entre interior y exterior) } \\
\mathrm{K} & =3,9 \mathrm{kcal} / \mathrm{m}^{2} \cdot \mathrm{h} ; \\
\mathrm{Q} & =180 \mathrm{kcal} / \mathrm{m}^{2} \cdot \mathrm{h} .
\end{aligned}
$$

\section{CALOR REQUERIDO PARA CALENTAR LA MASA HASTA 50 $0^{\circ} \mathrm{C}$}

Aplicando la fórmula:

en la cual:

$$
\mathrm{Q}=\Delta \mathrm{t} \cdot \mathrm{C}_{\mathrm{p}} \cdot \mathrm{p} \cdot \mathrm{d}
$$

$$
\begin{aligned}
\mathrm{p} & =\text { densidad } 2.300 \mathrm{~kg} / \mathrm{m}^{3} ; \\
\mathrm{d} & =\text { espesor de la losa: } 0,15 \mathrm{~m}, \mathrm{y} \\
\mathrm{C}_{\mathrm{p}} & =\text { calor específico: } 0,21 \mathrm{kcal} / \mathrm{kg} \cdot{ }^{\circ} \mathrm{C},
\end{aligned}
$$

obtenemos una potencia requerida total por $\mathrm{m}^{2}$ de: $3.032 \mathrm{kcal}$.

Indudablemente esta carga puede suponerse anulada en muchas horas; pero requiriendo este tratamiento térmico una temperatura uniforme, es conveniente que alcance esta temperatura de régimen en el menor tiempo posible. De esta forma, suponiendo que el período transitorio es sólo de 5 horas, deberemos considerar una potencia instalada de: $600 \mathrm{kcal} / \mathrm{h}$.

Hay que tener en cuenta que si la temperatura exterior nocturna es de $20^{\circ} \mathrm{C}$ el tiempo de puesta a régimen se reduce a unas 3 horas.

\section{CALOR PERDIDO POR EVAPORACION}

Como ya indicamos, las pérdidas debidas a evaporación, atenderán a la fórmula:

$$
\mathrm{Q}=0,6\left(\mathrm{~T}-\mathrm{T}^{\prime}\right)\left(1+\frac{\mathrm{V}}{1,16}\right) ;
$$

que en nuestro caso dará un valor de $60 \mathrm{kcal} / \mathrm{h}$ :

$$
\begin{aligned}
& \text { - para: } \mathrm{T}-\mathrm{T}^{\prime}=2^{\circ} \mathrm{C} ; \\
& \text { - para: } \mathrm{V}=5,8 \mathrm{~m} / \mathrm{s} .
\end{aligned}
$$


Además habrá que tener en cuenta las pérdidas por aportación de agua fresca, según la ecuación:

Resultando:

$$
\mathrm{Q}=\mathrm{W}^{\prime}\left(\mathrm{T}-\mathrm{T}_{1}\right) \cdot \mathrm{C}_{\mathrm{e}} \cdot
$$

$$
\mathrm{Q}=1,65 \mathrm{kcal} / \mathrm{h} \text {. }
$$

Indudablemente este valor deberá multiplicarse por un factor $f=12$, ya que esta aportación nunca será debidamente controlada.

En resumen, la carga que hay que considerar por este concepto será de: $80 \mathrm{kcal} / \mathrm{h}$.

PERdidas de TRANSMision POR EL SUELO

$$
\mathrm{Q}=179,4 \mathrm{kcal} / \mathrm{m}^{2} \cdot \mathrm{h} \text {. }
$$

\section{PERDIDAS POR INFILTRACION DE AIRE POR RENDIJAS}

$$
\begin{aligned}
& \mathrm{Q}=0,24 \frac{\mathrm{t}}{\mathrm{V}_{\mathrm{esp} .}} \times \text { volumen } \times \mathrm{n} .^{\circ} \text { renovaciones } ; \\
& \mathrm{Q}=0,285 \times 46 \times \frac{1}{2} ; \\
& \mathrm{Q}=26 \mathrm{kcal} / \mathrm{m}^{2} \cdot \mathrm{h} .
\end{aligned}
$$

\section{PERDIDAS DE TRANSMISION POR CERRAMIENTOS LATERALES}

$$
\mathrm{Q}=46 \times 1,5=69 \mathrm{kcal} / \mathrm{m}^{2} \cdot \mathrm{h} .
$$

Hemos supuesto un cerramiento exterior de madera doble, con aislante interior.

\section{POTENCIA CALORIFica TOTAL POR MODUlo}

- pérdidas en la pasta $\ldots \ldots=(80+600+180) \mathrm{kcal} \times 7,5(2,6+3,4+2,6)=55.470$;

- pérdidas en el suelo $\ldots \ldots=179,4 \mathrm{kcal} \times 7,5 \times 3,4=4.590$;

- pérdidas en cerramientos. $=69 \mathrm{kcal} \times 2 \times 3,4 \times 2,6=1.237$;

- pérdidas por infiltración $\ldots=26 \mathrm{kcal} \times 3,4 \times 7,5 \times 2,6=1.716$.

Resumen:

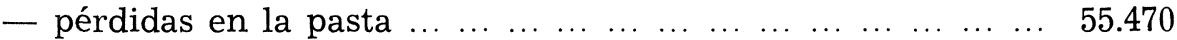

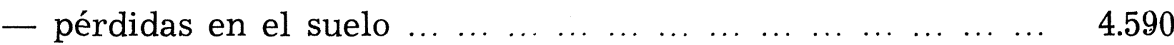

$$
\begin{aligned}
& \begin{array}{llllllllllllll}
- \text { pérdidas en cerramientos } & \ldots & \ldots & \ldots & \ldots & \ldots & \ldots & \ldots & \ldots & \ldots & \ldots & \ldots & 1.237
\end{array}
\end{aligned}
$$

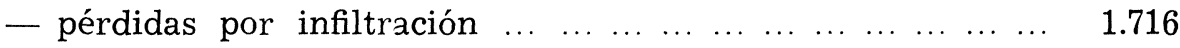

$$
\begin{aligned}
& \begin{array}{llllllll}
\text { TOTAL } & \ldots & \ldots & \ldots & \ldots & \ldots & \ldots & 63.013 \\
\mathrm{kcal} / \mathrm{h}
\end{array}
\end{aligned}
$$

Hay que destacar que no se han considerado las aportaciones debidas a las propias reacciones exotérmicas de fraguado. Puede considerarse este Capítulo como un margen de seguridad que nos disminuirá el tiempo de régimen.

Puede apreciarse cómo los tres últimos términos, aproximadamente un $12 \%$ del total, no se producirían con calefacción eléctrica. Estas pérdidas son muy inferiores en porcentaje al incremento de coste que supone utilizar aquel combustible. 